\title{
The Subconscious Director: Dynamically Personalizing Videos Using Gaze Data
}

\author{
Melanie Heck \\ University of Mannheim \\ Mannheim, Germany \\ melanie.heck@uni-mannheim.de \\ Jonathan Bünemann \\ University of Mannheim \\ Mannheim, Germany
}

\begin{abstract}
Watching TV has become a side event rather than a deliberate pastime. Movie directors thus struggle to find new ways to sustain the attention of their audience. Interactive movies usually require the viewer to actively decide how the plot progresses, creating an experience more akin to video games than film. In this paper, we propose a system that analyses gaze data to personalize the plot of a video without the viewer's active intervention. User preferences are inferred from their gaze allocation to different elements in a scene. The subsequent scene is then dynamically tailored towards the user's predicted preference. In a user study $(\mathrm{N}=175)$, we evaluate the effectiveness of the system with regard to user engagement. Our findings show that personalized videos have a positive effect on focused attention and involvement, whereas novelty perception is not significantly affected.
\end{abstract}

\section{CCS CONCEPTS}

- Human-centered computing $\rightarrow$ Human computer interaction (HCI); Interaction design process and methods; Contextual design.

\section{KEYWORDS}

eye tracking, adaptive media, gaze-contingent systems, preference prediction.

\section{ACM Reference Format:}

Melanie Heck, Janick Edinger, Jonathan Bünemann, and Christian Becker. 2021. The Subconscious Director: Dynamically Personalizing Videos Using Gaze Data. In 26th International Conference on Intelligent User Interfaces (IUI '21), April 14-17, 2021, College Station, TX, USA. ACM, New York, NY, USA, 11 pages. https://doi.org/10.1145/3397481.3450679

Permission to make digital or hard copies of all or part of this work for personal or classroom use is granted without fee provided that copies are not made or distributed for profit or commercial advantage and that copies bear this notice and the full citation on the first page. Copyrights for components of this work owned by others than the author(s) must be honored. Abstracting with credit is permitted. To copy otherwise, or republish, to post on servers or to redistribute to lists, requires prior specific permission and/or a fee. Request permissions from permissions@acm.org.

IUI '21, April 14-17, 2021, College Station, TX, USA

(C) 2021 Copyright held by the owner/author(s). Publication rights licensed to ACM ACM ISBN 978-1-4503-8017-1/21/04 ..\$15.00

https://doi.org/10.1145/3397481.3450679

\author{
Janick Edinger \\ University of Mannheim \\ Mannheim, Germany \\ janick.edinger@uni-mannheim.de \\ Christian Becker \\ University of Mannheim \\ Mannheim, Germany \\ christian.becker@uni-mannheim.de
}

\section{INTRODUCTION}

Imagine coming home from a long day at work and eventually finding the time for watching a movie. Having been truly captivated by the movie, you tell your co-worker about it and urge her to watch it. The next day, she thanks you for the recommendation. You are surprised to hear that she was intrigued by the victim's construction of an escape plan, but seems to have missed the part where the villain prepared the lethal coup. As you continue discussing the movie you notice that your co-worker's elaborations on some scenes do not reflect how you remember the plot. What happened here is no coincidence, but is really the making of the movie which allowed you to direct it yourself as you were watching the film. Without deliberately taking an action, you subconsciously decided about each turn in the plot while the movie evolved.

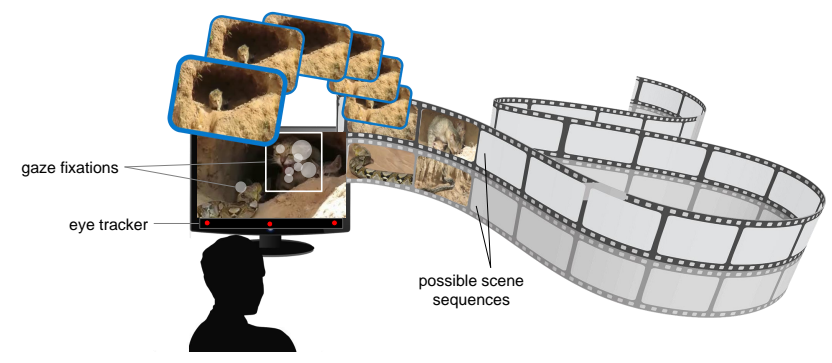

Figure 1: Illustration of a gaze-based adaptive movie. The next scene is dynamically selected based on the viewer's allocation of gaze to the objects in the current scene.

This futuristic scenario that changes the way we watch videos and movies could soon become reality. In 2018, Netflix launched the interactive movie "Black Mirror: Bandersnatch", where viewers make choices about subsequent scenes [59]. As the movie branches out into alternative continuations, viewers may arrive at five different endings, depending on their choices. Interactive documentaries such as iOtok [13] encourage viewers to interact with the featured episodes by clicking on supplementary material or discussing the topic with other viewers. While this allows viewers to control what they see, having them actively select their preferred plot or content conflicts with the purpose of movies, which is to provide laid-back 
entertainment. We thus propose twisting the storyline to the viewers' taste without them becoming actively engaged. We turn the viewer into the subconscious director of the movie.

We developed eyeDirect, a system for creating videos with an adaptive storyline based on gaze data. An eye tracker records the viewer's gaze as they watch a video sequence. Making use of the correlation between gaze allocation and preference for the fixated video object, eyeDirect selects a variant of the subsequent scene which features the viewer's preferred object (see Figure 1).

We make the following contributions:

- We present eyeDirect, a gaze-contingent system for adaptive video delivery. The system uses commercial products, and can thus be implemented using readily available technology.

- We conduct a user study $(\mathrm{N}=175)$ to analyze the effect of different personalization strategies on the user engagement (UE) attributes focused attention, involvement, and novelty.

The eyeDirect system may serve as a reference architecture for designing adaptive videos based on eye tracking data.

\section{RELATED WORK}

Gaze-contingent systems presuppose that preferences can be inferred from gaze. Evidence for a correlation between visual attention and preference for elements was already found in the 1960s. Mackworth and Morandi [36] report that important aspects of pictures receive more fixations. Engelke and Le Callet [16] identify a strong correlation between the two concepts. Yet, attention does not necessarily imply preference for an element. While topdown attention is goal-driven, i.e., it is controlled by the person [6], bottom-up attention reacts to salient elements (e.g., bright colors). Nevertheless, citing the results of several psychological studies, Bednarik [2] asserts that a link between cognitive processes and gaze data can be assumed. Market research has long made use of this link to analyze the determinants of consumers' decision-making process $[8,12,14,23,41,45,68]$. While market research traditionally analyzes gaze data offline, eyeDirect aims to infer preferences dynamically to adapt the system. We thus proceed with an overview of adaptive recommender systems in general, and gaze-contingent systems in particular.

\subsection{Recommender Systems}

Personalization requires user feedback to determine customer preferences. Explicit user feedback is proactively submitted by the user (e.g., product ratings). It potentially alters the natural system usage [11], and preference predictions are only as good as the users' ability to express their preferences [17]. Implicit user feedback thus extracts information from natural interactions with the system. Online stores use transaction data to recommend relevant products [4, 29, 35, 40]. Mobasher et al. [40] personalize search query results based on past transactions. At Amazon [35], customers are recommended products that others usually buy together with the products in their shopping cart. Facebook [4] enables subscribed brands to issue advertisements to the account of users who show interest in a product on their website, as well as to other Facebook users with similar profiles.

Since past user data becomes stale at a fast pace and often does not represent the user's current preferences, dynamic approaches aim to determine the users' current psychological state [38]. Browsing data such as search queries $[32,33,55,64]$ or clickstream data $[11,19]$ have proven to deliver reliable preference predictions in interactive web applications. In less interactive applications such as video streaming, in contrast, feedback from browsing data is scarce. Gaze-contingent systems therefore analyze users' eye movements to predict their preferred elements on the user interface.

\subsection{Gaze-contingent Systems}

Using eye tracking to augment elements that a user looks at was first proposed by Bolt [5] in 1981. Several muted video streams are simultaneously displayed on a large screen. Whenever the user looks at a stream for multiple seconds, the window is enlarged and its soundtrack is played. Vertegaal et al. [65] present a video communication system which only replays the speech output of whoever the user is currently looking at. Starker and Bolt [57] designed a graphics world inspired by Saint-Exupéry's "The Little Prince" in which items that the user looks at are augmented. iTourist [49] is an interactive map which uses gaze data to dynamically personalize audio information on landmarks. Meißner et al. [39] display information for products that users in a virtual reality shopping scenario are looking at.

Table 1: Related research on gaze-contingent systems

\begin{tabular}{|c|c|c|c|c|c|c|c|c|c|c|}
\hline & 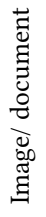 & $\underset{\substack{0 \\
i}}{\stackrel{8}{7}}$ & 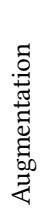 & 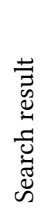 & 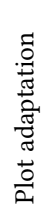 & 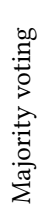 & 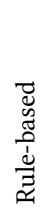 & 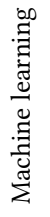 & 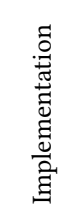 & 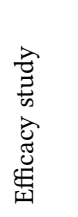 \\
\hline Reference & \multicolumn{2}{|c|}{ Subject } & \multicolumn{3}{|c|}{ Adaptation } & \multicolumn{3}{|c|}{ Prediction } & \multicolumn{2}{|c|}{ Contribution } \\
\hline [39] & $\bullet$ & & $\bullet$ & & & $\bullet$ & & & $\bullet$ & $\bullet$ \\
\hline [57] & • & & $\bullet$ & & & & $\bullet$ & & $\bullet$ & \\
\hline [49] & • & & $\bullet$ & & & & • & & $\bullet$ & $\bullet$ \\
\hline [10] & $\bullet$ & & & $\bullet$ & & & $\bullet$ & & - & $\bullet$ \\
\hline [30] & $\bullet$ & & & $\bullet$ & & & & $\bullet$ & $\bullet$ & \\
\hline [53] & $\bullet$ & & & $\bullet$ & & & & $\bullet$ & & \\
\hline [18] & $\bullet$ & & & $\bullet$ & & & & $\bullet$ & & \\
\hline [69] & & $\bullet$ & & $\bullet$ & & $\bullet$ & & & $\bullet$ & \\
\hline [5] & & $\bullet$ & $\bullet$ & & & $\bullet$ & & & $\bullet$ & \\
\hline [65] & & $\bullet$ & $\bullet$ & & & $\bullet$ & & & $\bullet$ & \\
\hline [27] & & $\bullet$ & $\bullet$ & & & & & $\bullet$ & & \\
\hline [47] & & $\bullet$ & & & $\bullet$ & & $\bullet$ & & & \\
\hline [66] & & $\bullet$ & & & $\bullet$ & $\bullet$ & $\bullet$ & & $\bullet$ & \\
\hline eyeDirect & & $\bullet$ & & & $\bullet$ & $\bullet$ & & $\bullet$ & $\bullet$ & $\bullet$ \\
\hline
\end{tabular}

Gaze-based recommender system may use browsing data to extract relevant images $[10,30]$, or predict document relevance from users' eye movements while reading text $[18,53]$. Xu et al. [69] additionally personalize recommendations for videos. They measure attention to key frames of a scene, and then predict attention to unseen videos with similar key frames. In contrast to our approach, attention is measured on the granularity of entire videos, instead of individual elements in a scene. Kandemir et al. [27] rank the relevance of elements in a video frame based on ordinal logistic 


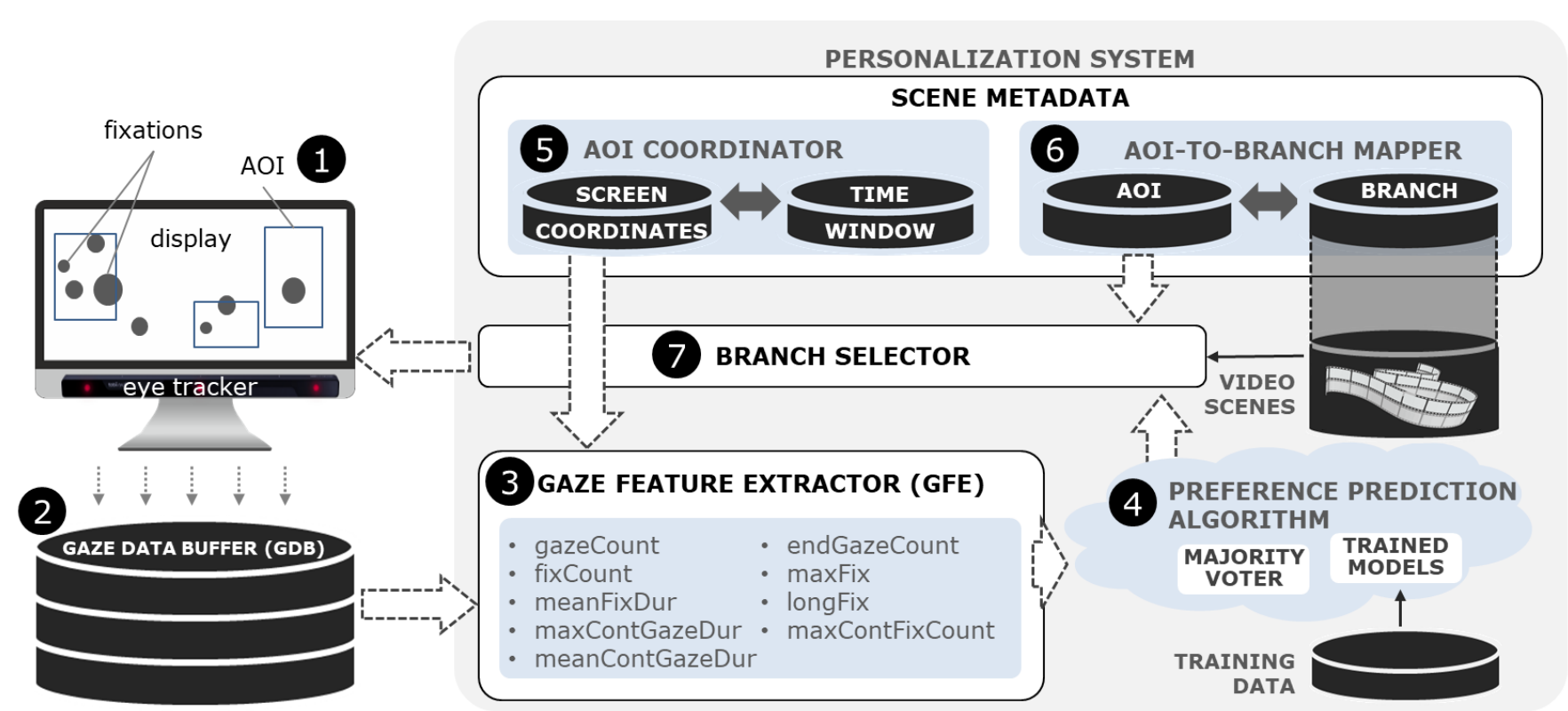

Figure 2: The eyeDirect system architecture for personalized video provisioning. The system analyzes real time gaze data to predict user preferences. Based on the predicted preference the system selects the next scene for the video.

regression. However, they do not apply the inferred preferences to personalize the video.

Hansen et al. [47] were the first to suggest using eye tracking for movies with an adaptive storyline. They describe a scene featuring two people who leave a room in opposite directions. The person to whom the viewer pays more attention is shown in the next scene. While the authors describe the concept for dynamic storyline adaptation, no implementation is presented. Vesterby et al. [66] propose a framework for gaze-based interactive movies. They build a prototype which predicts the viewer's preferred continuation of a scene, but do not assess the effectiveness of the system.

Table 1 summarizes existing gaze-contingent systems. None of the reviewed systems has both implemented a system for and evaluated the usability of adaptive movies. This paper thus presents eyeDirect, a system for dynamic video adaptation and assesses the effect of different personalization strategies on user engagement.

\section{THE EYEDIRECT SYSTEM}

EyeDirect is a system for creating adaptive movies using standard eye tracking equipment and machine learning models. The system analyzes gaze data collected at runtime and selects the next scene based on the viewer's preference (see Figure 2). It may serve as a reference architecture for creating gaze-based adaptive videos.

\subsection{Gaze Data Collection}

Gaze data is recorded during sequences in which all Areas of Interest (AOI) 1 are visible. An AOI is a rectangular region containing an element about which the system makes an inference. It may encompass one video of a tiled screen, or a single object like a person. AOI definition for moving elements can be automated with object tracking (e.g., [67]). A screen-based eye tracker starts recording the viewer's gaze at the beginning of a scene and continuously forwards the gaze data to the Gaze Data Buffer (GDB) 2. The GDB stores the raw gaze data for the entire scene. Gaze data collection stops two seconds before the scene ends, ensuring that data processing does not delay the display of the next scene.

\subsection{Feature Extraction}

At the end of each scene, the Gaze Feature Extractor (GFE) 3 processes the raw data accumulated in the GDB into a standard set of gaze features. Applying the strict average method [44], invalid gaze recordings are discarded, and the gaze coordinates of the left and right eye are averaged. The gaze points are then processed into fixations and saccades. During fixations, the viewer focuses on a specific point on the screen. We defined fixations based on the values recommended by Tobii [44] as eye movements with a velocity below a visual angle of $30 \%$. They are typically associated with the acquisition of information and can thus be leveraged for preference predictions $[10,24,37]$. During the saccadic jumps between fixations, in contrast, only little information is absorbed [26]. Most gaze features thus filter out saccades and only further process the extracted fixations. We extracted nine features that are commonly used in gaze-contingent systems:

(1) gazeCount: Total \# gaze points

(2) fixCount: Total \# fixations

(3) meanFixDur: Mean duration of fixations

(4) maxContGazeDur: Maximum continuous gaze time

(5) meanContGazeDur: Mean continuous gaze time

(6) endGazeCount: Gaze points during the last $400 \mathrm{~ms}$

(7) maxFix: Duration of the longest fixation

(8) longFix: \# fixations longer than $400 \mathrm{~ms}$

(9) maxContFixCount: Maximum \# continuous fixations 
The GFE then examines the distribution of gaze across the AOIs. Information about the screen coordinates of the AOIs is retrieved from the AOI Coordinator (5. Since the coordinates of a moving target may change within a scene, this module associates each AOI with its corresponding time window (defined in terms of milliseconds from the scene start).

\subsection{Preference Prediction}

The gaze features are forwarded to the Preference Prediction Algorithm 4 which determines the user's preferred AOI.

We integrate and test two prediction strategies: First, we use majority voting to analyze the distribution of a single feature over the AOIs. The viewer's preferred element is defined by the AOI with the highest value for the gaze feature.

Several properties of images including size, color, and placement of elements have been found to bias gaze allocation (e.g., [7],[15],[36]). In a second step, we therefore test whether training machine learning models with gaze data from subjects who have previously been shown the same scenes improves the effectiveness of the system.

\subsection{Branch Selection}

The Branch Selector 7 retrieves the video branch corresponding to the user's predicted preference. It issues a query to the AOI-toBranch Mapper 6. This module holds a tree-based record of all AOIs with their associated branch for the next scene. The mapping of AOIs to branches is a priori specified by the movie director. While this may result in a complex movie structure, interactive movies like "Black Mirror: Bandersnatch" have demonstrated that broadcasters possess both the resources and the capabilities to create such videos. Hansen et al. [66] suggest to maintain the complexity of the video manageable by embedding basing points into the system at which alternative branches converge.

When the scene ends, the Branch Selector projects the retrieved branch onto the display.

\section{SYSTEM EVALUATION}

The proposed eyeDirect system personalizes videos with the objective to increase user engagement. Existing research on web content personalization has suggested that users prefer personalized content and services $[21,60,63]$. We expect that a similar positive effect on user engagement can be achieved by personalizing videos $(\beta)$.

A second fundamental assumption of the system is that recording and processing a person's gaze data while watching a video allows to predict which elements the person prefers. The vision paper by Hansen et al. [47] suggests a rule-based adaptation. Personalized branching is initiated whenever gaze allocation to an object relative to other visible objects exceeds a certain threshold. The system for adaptive movies in [66] implements two rule-based adaptation strategies: The "winner-takes-it-all" strategy selects the object that correlates with the highest number of recorded gaze points. The "weight-decaying-interest" strategy assigns higher importance to gaze points that are recorded later in a scene. All these strategies are based on majority voting. Yet, their effect on user engagement has not been evaluated so far. In a first step, we therefore test the effectiveness of adapting videos based on a "winner-takes-it-all" majority voting $\left(\alpha_{1}\right)$.

State-of-the-art adaptive systems mostly use machine learning to predict user preferences [18, 27, 30, 53, 54]. While these models require prior scene-specific data collection, they are able to filter out saliency bias. We thus test whether machine learning adaptation is more effective than the majority voting that was proposed in the original systems $\left(\alpha_{2}\right)$.

When showing participants a continuation featuring only one element, any subsequent preference statements are likely to be biased. There is a good chance that subjects will choose the continued element as their preferred element simply because they were watching it for longer. We can thus not simultaneously evaluate whether a personalization strategy leads to accurate preference predictions $(\beta)$ and whether it indeed has an effect on user engagement $(\alpha)$. Instead, we evaluate how adapting a video based on to the predicted preference affects user engagement. We do not make any claims regarding the effectiveness of video personalization based on the viewer's true preferences. We test the following hypotheses:

- H1: Personalizing videos by applying majority voting to gaze features (naive prediction) increases user engagement.

- H2: Personalizing videos based on machine learning increases user engagement.

The personalization strategies are based on a causal model in which the relationship between preference predictions and user engagement is mediated by the preference-based personalization (see Figure 3).

We tested the hypotheses in a user study in which we recorded participants' gaze while they were watching three videos from the genres Music, Tutorial, and Documentary. An experimental betweensubjects design with one treatment group and one control group was adopted. In the treatment group, one out of two possible continuations of the video was chosen at random. Thus, some participants were shown a continuation featuring their preferred element in the video (positive personalization $P$ ). Others continued watching a

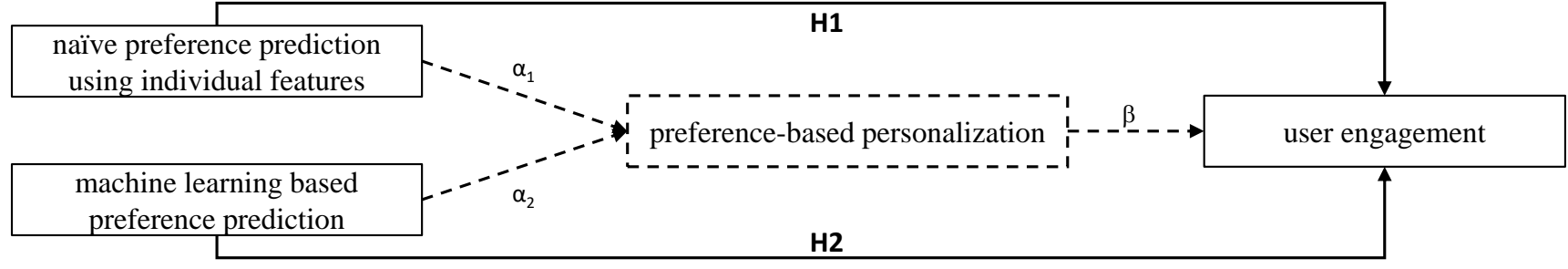

Figure 3: Causal model showing the hypothesized relationships between preference predictions and user engagement. 
(I)

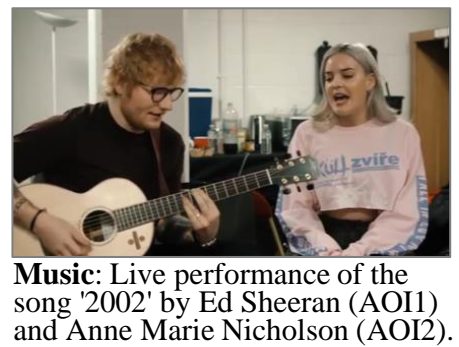

(II)

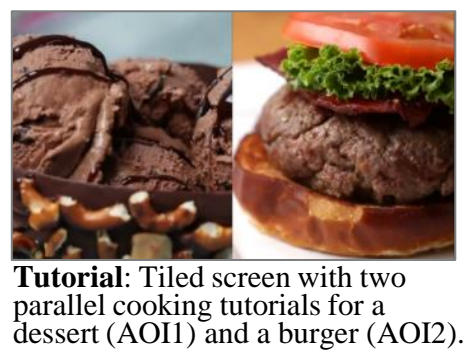

(III)

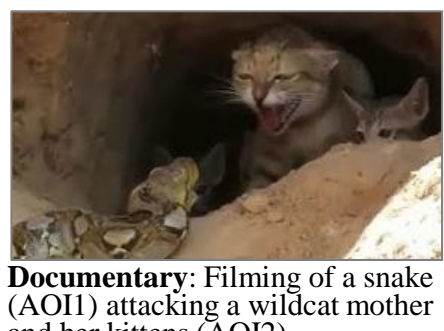

Figure 4: The experimental material consisted of 3 videos from the genres Music [42], Tutorial [61, 62], and Documentary [3].

scene showing their least preferred element (negative personalization $N$ ). In the control group, no branching was initiated. Instead, subjects were shown a generic $(G)$ continuation featuring both video elements. We then compared participants' user engagement scores when watching a video with positive personalization to (1) negative personalization, and (2) a non-adaptive generic video.

\subsection{Participants}

175 members and visitors of our research institution (93 male, mean age $=25.35, \sigma=8.39$ ) participated in the study. The experiment took about 5 minutes for each participant. Subjects were assigned quasirandomly to an experimental group according to the date of their arrival. They were informed that their gaze was being monitored, but they were not aware of its use for dynamic video adaptation.

\subsection{Apparatus}

The software ran on a $2.5 \mathrm{GHz}$ Intel Core i7-6500 HP PC. The PC drove a 23.6 inch iiyama touch monitor (1920 x 1080 pixels) on which the videos were displayed. Gaze data was collected with a Tobii Pro X3-120 eye tracker that was mounted to the display. The experiment was performed in our research lab. Participants were seated in front of the display, maintaining a distance between their eyes and the eye tracker of 20-30 inches.

\subsection{Experimental Material}

The experiment consisted of three videos from the genres $\mathrm{Music}, \mathrm{Tu}$ torial, and Documentary to reflect the content commonly available on video streaming platforms (see Figure 4).

Each video features two main elements which were defined as AOIs. We used two fullscreen videos (I, III) and one tiled video (II). The latter shows two video streams in parallel. Such a design allows, for example, to simultaneously present multiple trailer previews on television or on online video streaming platforms. An adaptive system may subsequently display a teaser scene of the movie that received the most attention.

\subsection{Procedure}

At the beginning of the experiment, a 5-point eye tracker calibration was performed. Subjects had to fixate five red circles that successively appeared on the screen. Completing the procedure required successful eye detection. If the eye tracker did not detect the subject's eyes, its orientation was adjusted. This ensured that gaze data was collected for all participants.
Participants were instructed to sit in a comfortable position and watch three video sequences. By granting subjects freedom of movement, we receive more realistic estimates of the system's performance in an uncontrolled setting. The order in which the sequences were displayed was identical for all participants. This experimental setup was chosen to maximize the uniformity of conditions between the experimental groups.

Gaze was recorded during the first part of the video, which was identical for all groups. In the first sequence, subjects were shown a live performance of the song '2002' by Ed Sheeran and Anne Marie [42]. After 39 seconds, the video branched out into alternative scenes. The treatment group was shown a stream featuring a solo performance of one of the two singers. The control group continued watching the duet. The second sequence displayed two video streams of a cooking tutorial in parallel, one for a dessert [62] and one for a burger [61]. After 28 seconds, the treatment group was shown a video of another recipe for either one of the food categories. The control group was again shown two videos in parallel. The third sequence showed a snake attack on a wildcat family [3]. The video halted after 33 seconds, when the wildcat was just about to launch a counterattack on the snake. The treatment group was shown the fate of one opponent. Subjects assigned to the control group were shown the outcome of the fight and followed the paths of both animals.

Directly after watching each video, subjects were presented an on-screen questionnaire asking them to rate their degree of engagement when watching the videos. Upon finishing the experiment, the accuracy of the gaze recordings was validated. Each subject was asked to fixate five circles which appeared in each corner and in the center of the display. They were then presented another questionnaire asking them to recall their preferred element for each of the three videos.

\subsection{Metrics}

We adapted O'Brian and Toms' User Engagement Scale [43] to measure user engagement when watching videos. Specifically, we asked participants to indicate their degree of agreement on a 6-point Likert scale for the following items:

- Q1: I'd like to watch the video for another minute. (novelty)

- Q2: I found the video interesting. (novelty)

- Q3: I was very attentive.

- Q4: I found the video enjoyable. (involvement) 


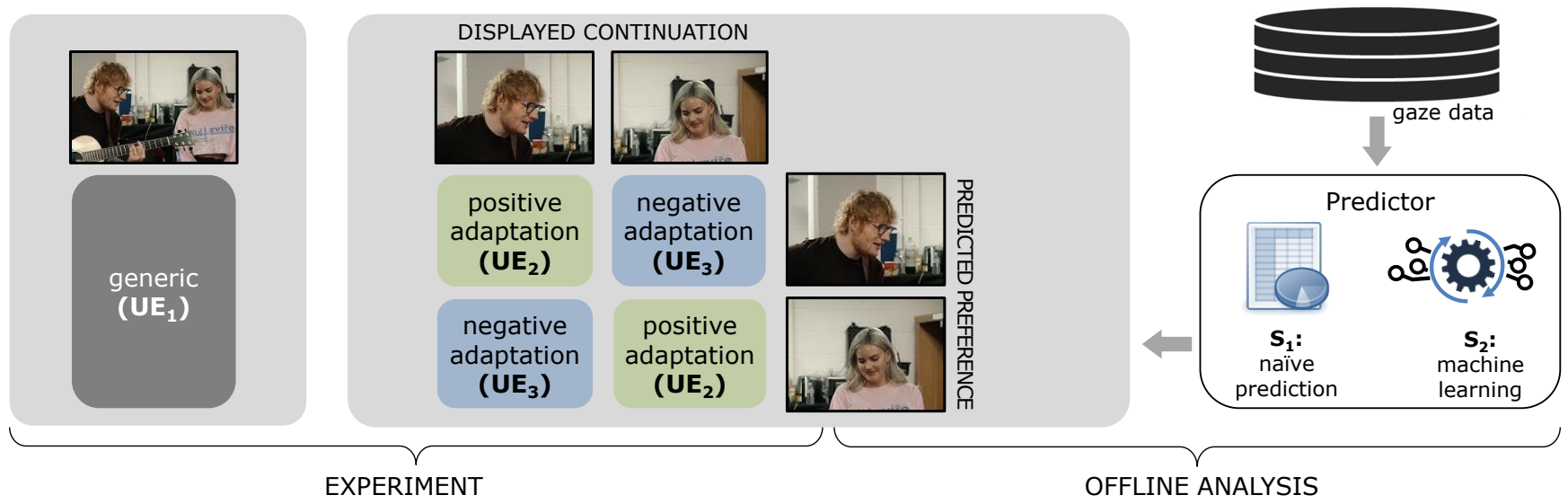

Figure 5: Illustration of the procedure to divide the samples into experimental groups. Samples where the predicted preference matches (does not match) the displayed continuation were assigned to the positive (negative) personalization group.

The first two items $(\mathrm{Q} 1, \mathrm{Q} 2)$ measure the attribute novelty. O'Brian and Toms define this attribute as a "variety of sudden and unexpected changes [...] that cause excitement and joy or alarm" [43]. The third item (Q3) is a measure of focused attention, which implies "concentrating on one stimulus only and ignoring all others" [43]. Q4 measures involvement, defined as a "need-based [...] psychological identification with some object" [43].

\section{RESULTS}

After excluding 29 samples due to insufficient quality of gaze recordings, we retained 146 samples. Gaze accuracy of the remaining samples was sufficient for assigning the data to an $\mathrm{AOI}$ (mean error $<.9^{\circ}$ visual angle). 51 subjects pertained to the control group. The other 95 subjects had been exposed to either positive or negative video personalization. In an offline evaluation, we simulate two personalization strategies and assess their effect on user engagement:

- S1: Naive Prediction. We predict the viewer's preferred element in the video by applying majority voting to individual gaze features. Based on this prediction, we categorize the samples into two groups. Subjects who are assigned to the positive personalization group had been shown the video continuation that matches their predicted preference. Subject of the negative personalization group had been shown the continuation that does not match their predicted preference. Finally, we compare the user engagement scores resulting from positive personalization to those from negative personalization and to the generic control group.

- S2: Machine Learning Prediction. Machine learning might be able to capture interdependencies between video elements and systematic biases towards a salient element. We thus examine whether adaptation with machine learning has a stronger effect on user engagement than the naive approach. We now predict the viewer's preferred element in the video using machine learning classifiers. This might result in a different assignment of subjects to the positive or negative personalization group. The effectiveness of personalization with machine learning predictions is then assessed by again comparing the UE scores between the experimental groups.

Figure 5 illustrates how subjects were assigned to their corresponding experimental group.

\subsection{S1: Naive Prediction}

In a first step, we predict the viewers' preference based on individual gaze features. This strategy selects the AOI for which the feature returns the highest value, computed for a participant. The main advantage of naive prediction is that no further information is required to make the decision (in contrast to machine learning which requires prior data collection). Subjects are assigned to either the positive or the negative personalization group depending on whether, based on the prediction, they had been shown a scene with their preferred element or not. Finally, we calculate the average UE score for each group (including the generic control group) as the mean score from all four UE measures.

The resulting effect on user engagement is inconsistent across the gaze features (see Figure 6). While six features (gazeCount, fixCount, meanFixDur, maxContGazeDur, endGazeCount, maxFix) make predictions that result in higher UE scores for the positive personalization group compared to the generic control group, the effect is reversed when predicting preferences with the remaining three features. Compared to the negative control group, only four features (gazeCount, meanFixDur, maxContGazeDur, maxFix) deliver higher UE scores. A one-sided t-test reveals that the naive strategy has no significant positive effect on user experience at any common level of confidence, independent of the underlying gaze feature. Table 2 details the difference in UE scores between the experimental groups for each gaze feature.

Based on these numbers, we reject $\mathbf{H 1}$. Two possible factors may be the cause for this result: Either the naive prediction strategy results in inaccurate preference predictions $\left(\alpha_{1}\right)$. or video personalization has no effect on UE $(\beta)$.

The data from the treatment group does not allow us to determine whether inaccurate preference predictions are the primary cause. 


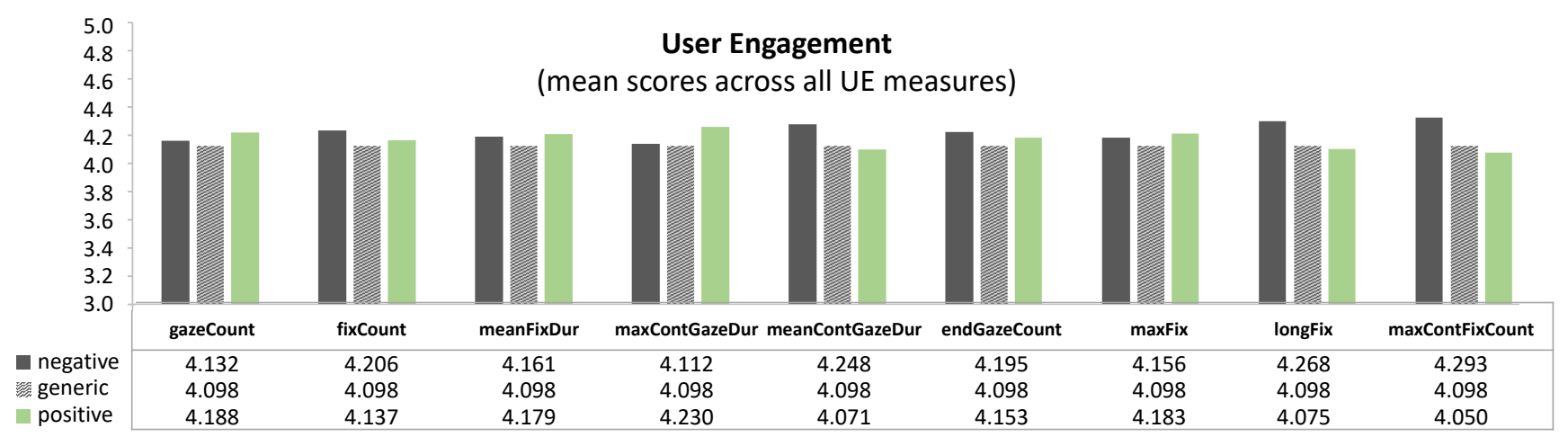

Figure 6: Comparison of UE between feature-based experimental groups.

Table 2: Impact of feature-based video personalization on UE

\begin{tabular}{|c|c|c|c|c|c|c|c|c|c|}
\hline & \multicolumn{9}{|c|}{ FEATURE } \\
\hline & {$[1]$} & {$[2]$} & {$[3]$} & {$[4]$} & {$[5]$} & {$[6]$} & [7] & [8] & [9] \\
\hline$\Delta \mathrm{UE}(\mathbf{P}-\mathbf{N})$ & .057 & $(-.069)$ & .018 & .118 & $(-.177)$ & $(-.042)$ & .027 & $(-.193)$ & $(-.243)$ \\
\hline p-value & .361 & .679 & .451 & .213 & .880 & .611 & .427 & .904 & .950 \\
\hline$\Delta \mathbf{U E}(\mathbf{P}-\mathbf{G})$ & .090 & .039 & .081 & .132 & $(-.027)$ & .055 & .085 & $(-.023)$ & $(-.048)$ \\
\hline $\mathrm{p}$-value & .261 & .399 & .297 & .195 & .566 & .354 & .287 & .561 & .622 \\
\hline
\end{tabular}

Difference in UE scores between experimental groups: positive personalization (P), negative personalization (N), generic sequence $(\mathrm{G})$.

No tested gaze feature delivers preference predictions that significantly improve UE.

This is due to the fact that personalized video delivery likely results in biased a posteriori preference statements of the study participants. Subjects of the control group, in contrast, have been exposed to all video elements to the same degree. Their reported preference statements from the follow-up questionnaire are therefore not corrupted by varying exposure times. We thus apply majority voting to the gaze features extracted from the control group and predict the viewers' preferred element in each video. We then compare the predicted preference to the subject's reported preference. The mean prediction accuracy from all videos ranges from $45.10 \%$ (meanContGazeDur) to $66.67 \%$ (endGazeCount). These accuracy values, which are even below the random baseline of $50 \%$ for some features, confirm that the ineffectiveness of the naive strategy can (at least in part) be attributed to inaccurate preference predictions. We thus cannot sustain assumption $\alpha_{1}$ (i.e., majority voting allows to predict user preferences). We proceed to investigate whether machine learning is more effective than the majority voting strategy proposed by the original adaptive movies.

\subsection{S2: Machine Learning Prediction}

We evaluate whether machine learning can more accurately adapt the video to the viewer's preferences. The classifiers are trained exclusively with control group data where no bias was induced as a consequence of prolonged exposure to an element. We selected five classifiers from the Python scikit-learn library [48] which are commonly used to infer user preferences: Logistic regression (Logit) [27, 30, 56], Passive Aggressive (PA) [56], k-Nearest Neighbor (k-NN) [28, 51, 52], Decision Tree (Tree) [29, 52], and Support Vector Classifier (SVC) [18, 22, 25, 50, 53, 56]. We identify the best feature subset by training each classifier with all possible combinations of gaze features on a randomly selected subset of the training data (see Table 3).

Table 3: Best feature subsets for machine learning classifiers

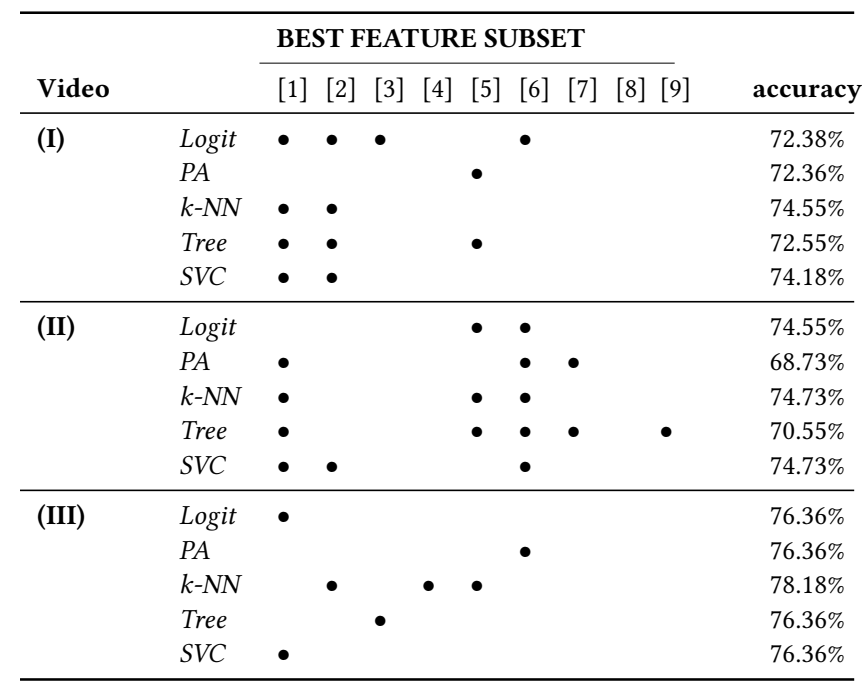

The models are trained for each video individually. This allows them to take into account that some video elements draw attention due to a salient property, and not as a result of the viewer's preference. Prediction accuracies from five-fold cross validation range 

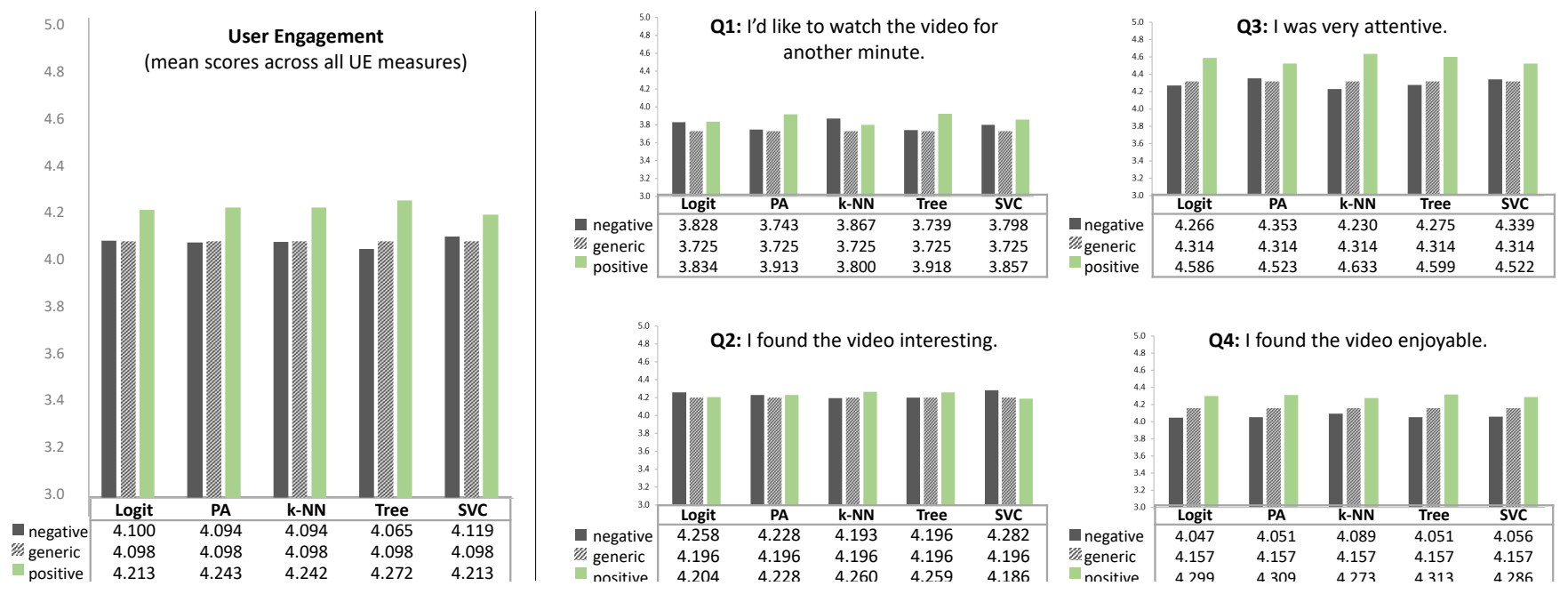

Figure 7: Comparison of overall and individual UE measures between ML-based experimental groups.

from $68.73 \%$ to $78.18 \%$. Although a prediction error of about $25 \%$ persists, all classifiers deliver significantly better predictions than both the random baseline and majority voting. We thus proceed to analyze whether, allowing for the remaining prediction error, eyeDirect is capable of increasing UE. The following predictions are based on the best feature subset for each classifier.

5.2.1 Overall User Engagement Evaluation. In order to derive generalizable conclusions, the following analyses are based on the aggregated results from all videos. We predict a subject's preferred video element by applying each classifier separately to the gaze data of the treatment group. Subjects are then again assigned to an experimental group based on whether the video had continued for them with their preferred element or not. We determine the mean UE score for each group and analyze whether significant differences exist between the experimental groups (see Figure 7).

The largest improvement in UE is achieved with the Tree classifier. Compared to negative personalization, an improvement of .207 points exists. The effect is significant at $90 \%$ confidence ( $\mathrm{p}$ value $=.080)$. Compared to the generic video display, UE scores from positive personalization are on average .174 points higher than for the generic group. Yet, the improvement is not significant at any common significance level ( $\mathrm{p}$-value $=.123$ ). We thus proceed to analyze each UE measure separately to verify whether video personalization has a positive effect on any individual UE measure.

5.2.2 Item-Specific User Engagement Evaluation. Using the group allocation from the overall UE evaluation, we calculate their UE scores for each of the four UE measures individually (see Figure 7). With almost all classifiers, positive personalization visibly improves focused attention (Q3) and involvement (Q4) compared to both negative personalization and generic videos. Involvement is significantly improved compared to the negative personalization group when applying the Logit $($ delta $=.252$, p-value $=.078)$, PA $($ delta $=.257, \mathrm{p}$ value $=.076)$, or Tree classifier $($ delta $=.262$, $\mathrm{p}$-value $=.071)$. Focused attention is improved compared to both negative personalization and generic video display. The effect is significant for Logit (P: delta $=.320, \mathrm{p}$-value $=.028 ; \mathrm{G}:$ delta $=.272, \mathrm{p}$-value $=.049), \mathrm{k}-\mathrm{NN}$ $(\mathrm{P}:$ delta $=.404, \mathrm{p}$-value $=.008 ; \mathrm{G}$ : delta $=.320$, $\mathrm{p}$-value $=.029)$, and Tree $(\mathrm{P}:$ delta $=.323, \mathrm{p}$-value $=.026 ; \mathrm{G}$ : delta $=.285$, $\mathrm{p}$-value $=.043)$.

The effect of positive personalization on the novelty measures (Q1, Q2), in contrast, is less poignant and is even negative in some of the evaluated scenarios. A one-sided t-test proves that none of the classifiers personalizes the videos in a way that has a significant positive impact on the viewers' novelty perception. Table 4 contrasts the mean effect of positive personalization on each UE measure to the results from the other experimental groups.

We thus confirm $\mathbf{H} 2$ for the user engagement attributes focused attention and involvement, but reject $\mathbf{H} 2$ for the attribute novelty. In view of the underlying principle of the eyeDirect system, these results are not surprising: The video is personalized by turning the element which the viewer had previously looked at the most into the protagonist. When applying negative personalization, in contrast, viewers are shown the element to which they had previously not paid much attention. This element may therefore hold more novelty than the element to which the viewer had already paid close attention. Focused attention and involvement, on the other hand, are more pronounced for elements to which the viewer has an emotional connection. Displaying the viewer's preferred element thus results in higher scores for these attributes.

\section{DISCUSSION}

We demonstrated that the eyeDirect system is able to increase user engagement while watching a video by spontaneously tailoring the plot to the viewer's predicted preferences. In the following, we discuss the validity of our findings, as well as their implications for developing systems that use gaze data to dynamically adapt videos.

\subsection{Validity of Gaze-based Predictions}

Using machine learning, eyeDirect was able to significantly increase the focused attention of a user watching a video. Compared to users who are shown a scene that does not match their preferences, subjects with positive personalization further experienced 
Table 4: Impact of ML-based video personalization on UE

\begin{tabular}{|c|c|c|c|c|c|}
\hline \multirow[b]{2}{*}{ User Engagement Item } & \multirow[b]{2}{*}{ Classifier } & \multicolumn{2}{|c|}{$\Delta($ UE SCORE $)$} & \multicolumn{2}{|c|}{ P-VALUE } \\
\hline & & $\mathbf{P}-\mathbf{N}$ & P-G & $\Delta(\mathbf{P}, \mathbf{N})$ & $\Delta(\mathbf{P}, \mathbf{G})$ \\
\hline \multirow[t]{5}{*}{ TOTAL } & Logistic Regression & .131 & .133 & 0.1883 & 0.1851 \\
\hline & Passive Aggressive & .150 & .145 & 0.1562 & 0.1648 \\
\hline & $\mathrm{k}-\mathrm{NN}$ & .147 & .144 & 0.1591 & 0.1714 \\
\hline & Decision Tree & .207 & .174 & $0.0804^{*}$ & 0.1230 \\
\hline & SVC & .094 & .115 & 0.2640 & 0.2192 \\
\hline \multirow[t]{5}{*}{ Q1: I'd like to watch the video for another minute. } & Logistic Regression & .006 & .109 & 0.4868 & 0.2781 \\
\hline & Passive Aggressive & .170 & .187 & 0.1839 & 0.1559 \\
\hline & $\mathrm{k}-\mathrm{NN}$ & $(-.067)$ & .075 & 0.6381 & 0.3455 \\
\hline & Decision Tree & .179 & .193 & 0.1709 & 0.1509 \\
\hline & SVC & .059 & .132 & 0.3789 & 0.2352 \\
\hline \multirow[t]{5}{*}{ Q2: I found the video interesting. } & Logistic Regression & $(-.054)$ & .008 & 0.6216 & 0.4818 \\
\hline & Passive Aggressive & .000 & .032 & 0.4994 & 0.4253 \\
\hline & $\mathrm{k}-\mathrm{NN}$ & .067 & .064 & 0.3492 & 0.3562 \\
\hline & Decision Tree & .063 & .062 & 0.3594 & 0.3581 \\
\hline & SVC & $(-.096)$ & $(-.010)$ & 0.7088 & 0.5231 \\
\hline \multirow[t]{5}{*}{ Q3: I was very attentive. } & Logistic Regression & .320 & .272 & $0.0282^{* *}$ & $0.0490^{* *}$ \\
\hline & Passive Aggressive & .171 & .210 & 0.1545 & 0.1047 \\
\hline & $\mathrm{k}-\mathrm{NN}$ & .404 & .320 & $0.0075^{* * *}$ & $0.0287^{* *}$ \\
\hline & Decision Tree & .323 & .285 & $0.0263^{* *}$ & $0.0431^{* *}$ \\
\hline & SVC & .183 & .208 & 0.1381 & 0.1048 \\
\hline \multirow[t]{5}{*}{ Q4: I found the video enjoyable. } & Logistic Regression & .252 & .143 & $0.0797^{*}$ & 0.1985 \\
\hline & Passive Aggressive & .257 & .152 & $0.0758^{*}$ & 0.1836 \\
\hline & $\mathrm{k}-\mathrm{NN}$ & .184 & .116 & 0.1512 & 0.2498 \\
\hline & Decision Tree & .262 & .156 & $0.0716^{*}$ & 0.1791 \\
\hline & SVC & .229 & .129 & 0.1016 & 0.2202 \\
\hline
\end{tabular}

Difference in UE scores between experimental groups: positive personalization (P), negative personalization (N), generic sequence $(\mathrm{G})$.

P-values of a one-sided t-test indicate which classifiers result in a significant improvement in UE (significance level: ${ }^{*} 10 \%,{ }^{* *} 5 \%,{ }^{* * *} 1 \%$ ).

higher involvement with the video. This finding will be relevant for situations in which a generic scene is not appropriate, and thus branching cannot be avoided. Instead of randomly choosing one branch, the director may offer several variations of the scene. The system then dynamically selects the user's preferred variant.

In prior work [20] we found that the system delivers accurate predictions for videos independent of their design. However, better predictions can be achieved when the viewer has a strong preference for one of the elements of interest. Simulations with the eyeDirect system showed that simple majority voting based on individual gaze features is not capable of delivering similar results. This suggests that salient elements bias the viewer's gaze, and raw gaze allocation therefore does not adequately represent a viewer's preferences. Machine learning filters out salient factors, but requires prior collection of scene-specific training data. In order to avoid repeating the time-consuming data collection for each new scene, Qvarfordt and Zhai [49] remove confounding factors by parameterizing their effect for each object. Cheng et al. [9] compare new scenes to a database of scenes for which training data has been collected. Machine learning classifiers are trained with the data of comparable scenes. The fitted model is then used to predict preferences for the new scene.

The significant positive effect on involvement and focused attention even for short videos reveals the potential of personalization for fully fledged movies. It may not only result in higher entertainment, but also be relevant when sustaining the audience's attention is critical such as in educational or safety videos.

Novelty, in contrast, did not increase for users who were shown a personalized video. We do, however, expect long-term effects on novelty perception for users who watch a video several times. In particular, when a movie has several branches, the plot may change when the user watches the movie repeatedly, leading to a completely new user experience.

\subsection{Use Case Implications}

We conceive multiple scenarios in which eyeDirect can be beneficial. First, viewers can enjoy a movie whose plot is tailored to their individual preferences without the need for active involvement. Since eyeDirect relies on the collection of conclusive eye tracking data, the movie director has to specify which branch selection rule is applied in the case of inconclusive gaze data: The system may display either a generic scene, or select a default branch. Alternatively, eyeDirect can be extended with further feedback mechanisms such as biosensor data or facial expressions. This allows to determine the viewer's degree of contentment with the current scene even if no accurate gaze data is collected.

Second, educational or instructional videos can become more appealing when they arouse the viewer's interest. A compelling 
example are safety instructions on airplanes where each passenger has their own screen. Airlines invest a lot of resources into these videos to gain the passengers' attention. A brief overture into such a video can elicit a passenger's preferred frame story and select one out of multiple versions of the safety video.

Third, personalized advertising typically requires historical transaction data. EyeDirect allows video streaming platforms to identify the momentary needs of viewers and display only advertisements that are currently relevant to them.

\subsection{Considerations for Consumer App Design}

Even though eye tracking is a mature technique that is used in multiple academic and non-academic contexts, some work is still needed to make the proposed system accessible to a greater audience. Accurate gaze estimations require user-specific calibration [37]. Especially when applying the system to short videos, the traditional five-point calibration would distract the user from a seamless viewing experience. Krafka et al. [31] show how deep learning might help to create a calibration-free system, but also recognize that quality of gaze estimates is considerably higher after calibration.

Additionally, eyeDirect has only been tested under controlled laboratory conditions. The high-quality Tobii Pro X3-120 eye tracker that we used in our studies further requires special hardware which is impractical and too expensive for a common user. Less sophisticated devices will lower the quality of the recorded gaze data and make preference predictions less accurate. This effect will be even stronger when coupled with the additional confounding factors that persist in real-world settings, such as changing lighting conditions, or users frequently diverting their gaze away from the screen. However, open-source solutions like OpenFace [1], GazeML [46], and MPIIFaceGaze [71] that use built-in device cameras are rapidly catching up in their performance and are becoming more resilient to changing environmental conditions. While accuracy is still lower than with specialized hardware, it is sufficient for assigning gaze coordinates to an AOI [70].

Our studies show that gaze data carries a wealth of information and might reveal personal dates such as political, religious, or sexual preferences. Users might therefore not be willing to grant camera access to the application. Among IoT applications, eye tracking provokes the strongest resistance, especially for preference identification tasks $[34,58]$. But since users are less concerned when their data is not forwarded to others [58], we expect less privacy concerns for eyeDirect: Gaze data is processed locally and can be discarded after the user's preference for an element has been determined. It is, however, the duty of the system designer to ensure responsible handling of the collected data.

\section{CONCLUSION}

We presented eyeDirect, a system for personalized video provisioning based on gaze data. In a comprehensive user study $(\mathrm{N}=175)$, we showed that personalized videos induce higher focused attention compared to videos with a generic storyline. Positive personalization also leads to higher involvement with the video compared to both negative personalization and generic videos. Novelty perception, in contrast, is not increased through the proposed system.
While simple majority voting with individual gaze features is not capable of adapting the system in a way that is meaningful to the user, personalization based on machine learning does have the desired impact on user engagement.

In future work, we plan to study how physiological sensor data can be used to improve the system. We expect that this will allow to infer the viewer's attitude towards an element even if only a single object is displayed at a time.

\section{REFERENCES}

[1] Tadas Baltrusaitis, Peter Robinson, and Louis-Philippe Morency. 2016. OpenFace: An open source facial behavior analysis toolkit. In WACV'16. IEEE. https://doi. org/10.1109/WACV.2016.7477553

[2] Roman Bednarik. 2005. Potentials of eye-movement tracking in adaptive systems. In $U M$ ' 05 .

[3] Best Of Wildlife. 2019. Brave moment cat fights a large python to protect its kittens. https://www.youtube.com/watch?v=2yY5gy0u4Ck

[4] Anthony Bigornia. 2015. IBM/Facebook partnership. Making irrelevant ads a thing of the past. https://www.ibm.com/blogs/insights-on-business/consumerproducts/ibmfacebook-partnership-making-irrelevant-ads-a-thing-of-thepast/

[5] Richard A. Bolt. 1981. Gaze-orchestrated dynamic windows. Computer Graphics 15, 3 (1981), 109-119.

[6] Raymond R. Burke and Alex Leykin. 2014. Identifying the drivers of shopper attention, engagement, and purchase. Review of Marketing Research 11 (2014), 147-187. https://doi.org/10.1108/S1548-643520140000011006

[7] Georg Buscher, Edward Cutrell, and Meredith Ringel Morris. 2009. What do you see when you're surfing?: Using eye tracking to predict salient regions of web pages. In CHI '09. ACM, 21-30. https://doi.org/10.1145/1518701.1518705

[8] Chun Tuan Chang and Pei Chi Chen. 2017. Cause-related marketing ads in the eye tracker: It depends on how you present, who sees the ad, and what you promote. International fournal of Advertising 36, 2 (2017), 336-355. https: //doi.org/10.1080/02650487.2015.1100698

[9] Shiwei Cheng, Xiaojian Liu, Pengyi Yan, Jianbo Zhou, and Shouqian Sun. 2010. Adaptive user interface of product recommendation based on eye-tracking. In EGIHMI '10. ACM, 94-101. https://doi.org/10.1145/2002333.2002348

[10] Shiwei Cheng and Ying Liu. 2012. Eye-Tracking Based Adaptive User Interface: Implicit Human-Computer Interaction for Preference Indication. Fournal on Multimodal User Interfaces 5, 1-2 (2012), 77-84. https://doi.org/10.1007/s12193011-0064-6

[11] Mark Claypool, Phong Le, Makoto Wased, and David Brown. 2001. Implicit interest indicators. In IUI '01. 33-40. https://doi.org/10.1145/359784.359836

[12] Jesper Clement, Tore Kristensen, and Kjell Grønhaug. 2013. Understanding consumers' in-store visual perception: The influence of package design features on visual attention. Journal of Retailing and Consumer Services 20, 2 (2013), 234-239. https://doi.org/10.1016/j.jretconser.2013.01.003

[13] Julie Ducasse, Matjaž Kljun, and Klen Čopič Pucihar. 2020. Interactive web documentaries: A case study of audience reception and user engagement on iOtok. International fournal of Human-Computer Interaction 36, 16 (2020), 15581584. https://doi.org/10.1080/10447318.2020.1757255

[14] Andrew T. Duchowski. 2002. A breadth-first survey of eye-tracking applications. Behavior Research Methods, Instruments, and Computers 34, 4 (2002), 455-470. https://doi.org/10.3758/BF03195475

[15] F. L. Engel. 1974. Visual conspicuity and selective background interference in eccentric vision. Vision Research 14, 7 (1974), 459-471. https://doi.org/10.1016/ 0042-6989(74)90034-0

[16] Ulrich Engelke and Patrick Le Callet. 2015. Perceived interest and overt visual attention in natural images. Signal Processing: Image Communication 39, B (2015), 386-404. https://doi.org/10.1016/j.image.2015.03.004

[17] Nikolaus Franke, Peter Keinz, and Christoph J. Steger. 2009. Testing the value of customization: When do customers really prefer products tailored to their preferences? fournal of Marketing 73, 5 (2009), 103-121. https://doi.org/10.1509/ jmkg.73.5.103

[18] David Hardoon, John Shawe-Taylor, Antti Ajanki, Kai Puolamäki, and Samuel Kaski. 2007. Information retrieval by inferring implicit queries from eye movements. In AISTATS '07, Vol. 2. PMLR, 179-186.

[19] John R. Hauser, Glen L. Urban, Guilherme Liberali, and Michael Braun. 2009. Website morphing. Marketing Science 28, 2 (2009), 202-223. https://doi.org/10. $1287 /$ mksc. 1080.0459

[20] Melanie Heck, Jonathan Bünemann, and Christian Becker. 2021. Exploring gaze-based prediction strategies for preference detection in dynamic interface elements. In CHIIR '21. ACM. https://doi.org/10.1145/3406522.3446013

[21] Shuk Ying Ho. 2006. The attraction of internet personalization to web users. Electronic Markets 16, 1 (2006), 41-50. https://doi.org/10.1080/10196780500491162 
[22] Eric Horvitz. 1999. Principles of mixed-initiative user interfaces. In $\mathrm{CHI}$ '99. ACM 159-166. https://doi.org/10.1145/302979.303030

[23] Yoon Min Hwang and Kun Chang Lee. 2018. Using an eye-tracking approach to explore gender differences in visual attention and shopping attitudes in an online shopping environment. International fournal of Human-Computer Interaction 34, 1 (2018), 15-24. https://doi.org/10.1080/10447318.2017.1314611

[24] Robert J. K. Jacob. 1991. The use of eye movements in human-computer interaction techniques: what you look at is what you get. ACM Transactions on Information Systems 9, 2 (1991), 152-169. https://doi.org/10.1145/123078.128728

[25] Thorsten Joachims, Laura Granka, Bing Pan, Helene Hembrooke, and Geri Gay. 2005. Accurately interpreting clickthrough data as implicit feedback. In SIGIR '05. ACM, 154-161. https://doi.org/10.1145/1076034.1076063

[26] Thorsten Joachims, Laura Granka, Bing Pan, Helene Hembrooke, and Geri Gay. 2017. Accurately interpreting clickthrough data as implicit feedback. SIGIR Forum 51, 1 (Aug. 2017), 4-11. https://doi.org/10.1145/3130332.3130334

[27] Melih Kandemir, Veli-Matti Saarinen, and Samuel Kaski. 2010. Inferring object relevance from gaze in dynamic scenes. In ETRA '10. ACM, 105-108. https: //doi.org/10.1145/1743666.1743692

[28] Przemysław Kazienko and Michał Adamski. 2007. AdROSA - Adaptive personalization of web advertising. Information Sciences 177, 11 (2007), 2269-2295. https://doi.org/10.1016/j.ins.2007.01.002

[29] Jong Woo Kim, Byung Hun Lee, Michael J. Shaw, Hsin Lu Chang, and Matthew Nelson. 2001. Application of decision-tree induction techniques to personalized advertisements on internet storefronts. International fournal of Electronic Commerce 5, 3 (2001), 45-62. https://doi.org/10.1080/10864415.2001.11044215

[30] László Kozma, Arto Klami, and Samuel Kaski. 2009. GaZIR: Gaze-based zooming interface for image retrieval. In ICMI '09. ACM, 305-312. https://doi.org/10.1145/ 1647314.1647379

[31] Kyle Krafka, Aditya Khosla, Petr Kellnhofer, Harini Kannan, Suchendra Bhandarkar, Wojciech Matusik, and Antonio Torralba. 2016. Eye tracking for everyone. In CVPR '16. IEEE, 2176-2184. https://doi.org/10.1109/CVPR.2016.239

[32] Tung Yen Lai and Gwo Haur Hwang. 2010. Application of half-life theory and fuzzy theory to a selection and recommendation system for web advertisement delivery in consideration of the time effect. WSEAS Transactions on Information Science and Applications 7, 1 (2010), 70-80.

[33] Marc Langheinrich, Atsuyoshi Nakamura, Naoki Abe, Tomonari Kamba, and Yoshiyuki Koseki. 1999. Unintrusive customization techniques for web advertising. Computer Networks 31, 11-16 (1999), 1259-1272. https://doi.org/10.1016/ S1389-1286(99)00033-X

[34] Hosub Lee and Alfred Kobsa. 2017. Privacy preference modeling and prediction in a simulated campuswide IoT environment. In PerCom '17. 276-285. https: //doi.org/10.1109/PERCOM.2017.7917874

[35] Greg Linden, Brent Smith, and Jeremy York. 2003. Amazon.com recommendations: Item-to-item collaborative filtering. IEEE Internet Computing 7, 1 (2003), 76-80. https://doi.org/10.1109/MIC.2003.1167344

[36] Norman H. Mackworth and Anthony J. Morandi. 1967. The gaze selects information details within picutres. Perception \& Psychophysics 2, 11 (1967), 547-552. https://doi.org/10.3758/BF03210264

[37] Päivi Majaranta and Andreas Bulling. 2014. Eye tracking and eye-based human computer interaction. In Advances in Physiological Computing, S. H. Fairclough and K. Gilleade (Eds.). Springer, London, UK.

[38] Sandra C. Matz and Oded Netzer. 2017. Using Big Data as a window into con sumers' psychology. Current Opinion in Behavioral Sciences 18 (2017), 7-12. https://doi.org/10.1016/j.cobeha.2017.05.009

[39] Martin Meißner, Jella Pfeiffer, Thies Pfeiffer, and Harmen Oppewal. 2019. Combining virtual reality and mobile eye tracking to provide a naturalistic experimental environment for shopper research. Fournal of Business Research 100 (2019), 445458. https://doi.org/10.1016/j.jbusres.2017.09.028

[40] Bamshad Mobasher, Robert Cooley, and Jaideep Srivastava. 2000. Automatic personalization based on web usage mining. Commun. ACM 43, 8 (2000), 142-151. https://doi.org/10.1145/345124.345169

[41] Francisco Muñoz-Leiva, Janet Hernández-Méndez, and Diego Gómez-Carmona. 2019. Measuring advertising effectiveness in Travel 2.0 websites through eyetracking technology. Physiology and Behavior 200, December 2017 (2019), 83-95. https://doi.org/10.1016/j.physbeh.2018.03.002

[42] Ed Sheeran Nicholson, Anne-Marie R. 2018. 2002. https://www.youtube.com/ watch? $=\mathrm{u} 3 \mathrm{ePPA} 0 \mathrm{yzSU}$

[43] Heather L. O'Brien and Elaine G. Toms. 2010. The development and evaluation of a survey to measure user engagement. Fournal of the American Society for Information Science and Technology 61, 1 (2010), 50-69. https://doi.org/10.1002/asi

[44] Anneli Olsen. 2012. The Tobii I-VT fixation filter. (2012), 21 pages.

[45] Tobias Otterbring, Erik Wästlund, Anders Gustafsson, and Poja Shams. 2014 Vision (im)possible? The effects of in-store signage on customers' visual attention. Journal of Retailing and Consumer Services 21, 5 (2014), 676-684. https://doi.org/ 10.1016/j.jretconser.2014.05.002

[46] Seonwook Park, Xucong Zhang, Andreas Bulling, and Otmar Hilliges. 2018. Learning to find eye region landmarks for remote gaze estimation in unconstrained settings. In ETRA '18. ACM. https://doi.org/10.1145/3204493.3204545
[47] John Paulin Hansen, Allan W. Andersen, and Peter Roed. 1995. Eye-gaze control of multimedia systems. Advances in Human Factors/Ergonomics 20, C (1995), 37-42. https://doi.org/10.1016/S0921-2647(06)80008-0

[48] Fabian Pedregosa, Gael Varoquaux, Alexandre Gramfort, Vincent Michel, Bertrand Thirion, Oliver Grisel, Mathieu Blondel, Peter Prettenhofer, Ron Weiss, Vincent Dubourg, Jake Vanderplas, Alexandre Passos, and David Cournapeau. 2011. Scikit-learn: Machine Learning in Python. Journal of Machine Learning Research 12 (2011), 2825-2830. https://doi.org/10.1007/s13398-014-0173-7.2

[49] Pernilla Qvarfordt and Shumin Zhai. 2005. Conversing with the user based on eyegaze patterns. In CHI '05. ACM, 221-230. https://doi.org/10.1145/1054972.1055004

[50] Filip Radlinski and Thorsten Joachims. 2005. Ouery chains: Learning to rank from implicit feedback. In KDD '05. ACM, 239-248. https://doi.org/10.1145/ 1081870.1081899

[51] Berthier Ribeiro-Neto, Marco Cristo, Paulo B. Golgher, and Edleno Silva de Moura. 2005. Impedance coupling in content-targeted advertising. In SIGIR '05. ACM, 496-503. https://doi.org/10.1145/1076034.1076119

[52] Ling Rothrock, Richard Koubek, Frederic Fuchs, Michael Haas, and Gavriel Salvendy. 2002. Review and reappraisal of adaptive interfaces: Toward biologically inspired paradigms. Theoretical Issues in Ergonomics Science 3, 1 (2002), 47-84. https://doi.org/10.1080/14639220110110342

[53] Jarkko Salojärvi, Kai Puolamäki, and Samuel Kaski. 2004. Relevance feedback from eye movements for proactive information retrieval. Technical Report. Helsinki University of Technology.

[54] Christina Schweikert, Louis Gobin, Shuxiao Xie, Shinsuke Shimojo, and D. Frank Hsu. 2018. Preference prediction based on eye movement using Multi-layer Combinatorial Fusion. In BI '18. Springer, 282-293. https://doi.org/10.1007/9783-030-05587-5_27

[55] Maad Shatnawi and Nader Mohamed. 2012. Statistical techniques for online personalized advertising. In SAC '12. 680. https://doi.org/10.1145/2245276.2245406

[56] Gino Slanzi, Jorge A. Balazs, and Juan D. Velásquez. 2017. Combining eye tracking, pupil dilation and EEG analysis for predicting web users click intention. Information Fusion 35 (2017), 51-57. https://doi.org/10.1016/j.inffus.2016.09.003

[57] India Starker and Richard A. Bolt. 1990. A gaze-responsive self-disclosing display. In CHI '90. ACM, 3-10. https://doi.org/10.1145/97243.97245

[58] Julian Steil, Inken Hagestedt, Michael Xuelin Huang, and Andreas Bulling. 2018. Privacy-aware eye tracking using differential privacy. CoRR abs/1812.0 (2018), 1-9. https://doi.org/10.1145/3314111.3319915

[59] Jackie Strause. 2018. 'Black Mirror' bosses unpack the multiple 'Bandersnatch' endings. https://www.hollywoodreporter.com/live-feed/black-mirrorbandersnatch-endings-explained-1171556

[60] Kar Yan Tam and Shuk Ying Ho. 2005. Web personalization as a persuasion strategy: An elaboration likelihood model perspective. Information Systems Research 16, 3 (2005), 271-291. https://doi.org/10.1287/isre.1050.0058

[61] Tasty. 2016. 4 burgers around the world. https://www.youtube.com/watch?v= Rix \{\} \}b9ThLI

[62] Tasty. 2019. 4 mind blowing ice cream tasty desserts. https://www.youtube. com/watch? $=$ VzHcBimEjh $8\{\&\} \mathrm{t}=76 \mathrm{~s}$

[63] Trang P. Tran. 2017. Personalized ads on facebook: An effective marketing tool for online marketers. Fournal of Retailing and Consumer Services 39 (2017), 230-242. https://doi.org/10.1016/j.jretconser.2017.06.010

[64] Michael Trusov, Liye Ma, and Zainab Jamal. 2016. Crumbs of the cookie: User profiling in customer-base analysis and behavioral targeting. Marketing Science 35, 3 (2016), 405-426. https://doi.org/10.1287/mksc.2015.0956

[65] Roel Vertegaal, Jeffrey S. Shell, Daniel Chen, and Aadil Mamuji. 2006. Designing for augmented attention: Towards a framework for attentive user interfaces. Computers in Human Behavior 22, 4 (2006), 771-789.

[66] Tore Vesterby, Jonas C. Voss, John Paulin Hansen, Arne John Glenstrup, Dan Witzner Hansen, and Mark Rudolph. 2005. Gaze-guided viewing of interactive movies. Digital Creativity 16, 4 (2005), 193-204. https://doi.org/10. 1080/14626260500476523

[67] Naiyan Wang and Dit-Yan Yeung. 2013. Learning a deep compact image representation for visual tracking. 26 (2013), 809-817.

[68] Michel Wedel and Rik Pieters. 2006. Eye tracking for visual marketing. Foundations and Trends in Marketing 1, 4 (2006). https://doi.org/10.1561/1700000011

[69] Songhua Xu, Hao Jiang, and Francis C.M. Lau. 2008. Personalized online document, image and video recommendation via commodity eye-tracking. In RecSys '08. ACM, 83-90. https://doi.org/10.1145/1454008.1454023

[70] Xucong Zhang, Yusuke Sugano, and Andreas Bulling. 2019. Evaluation of appearance-based methods and implications for gaze-based applications. In $\mathrm{CHI}$ '19. ACM, Article 416, 13 pages. https://doi.org/10.1145/3290605.3300646

[71] Xucong Zhang, Yusuke Sugano, Mario Fritz, and Andreas Bulling. 2017. It's written all over your face: Full-face appearance-based gaze estimation. In CVPR '17. IEEE, 51-60. https://doi.org/10.1109/CVPRW.2017.284 